\title{
Neue S2k-Leitlinie zur Tuberkulose im Erwachsenenalter
}

Derzeit befindet sich die S2k-Leitlinie (S2k - Konsens-basiert) „Tuberkulose im Erwachsenenalter - Leitlinie zur Diagnostik und Therapie einschließlich Chemoprävention und -prophylaxe“ im Druck. Die Leitlinie wurde durch das Deutsche Zentralkomitee zur Bekämpfung der Tuberkulose e.V. (DZK) im Auftrag der Deutschen Gesellschaft für Pneumologie und Beatmungsmedizin e.V. (DGP) erstellt. Unter Mitwirkung zahlreicher weiterer Fachgesellschaften wurde die im November 2014 angemeldete Leitlinie nach fünf Sitzungen zur Konsensfindung abgeschlossen (Details s. www.awmf.org/leitlinien/detail/anmeldung/1/ 11/048-o16.html). Die Therapieempfehlungen des DZK von $2012^{1}$ wurden durch diesen Prozess aktualisiert und auf ein AWMF-konformes Leitlinienniveau gebracht (AWMF - Arbeitsgemeinschaft der Wissenschaftlichen Medizinischen Fachgesellschaften).

Im Vergleich zu den Empfehlungen aus dem Jahr 2012 werden einige Empfehlungen zur Tuberkulosetherapie nun durch eigenständige Kapitel ausführlicher dargestellt. Hierzu gehören Patienten-Versorgungsaspekte, die Labordiagnostik und das therapeutische MedikamentenManagement. Die Kapitel zu Mehrfachresistenzen, HIVKoinfektionen und zu Arzneimitteln für die Tuberkulosetherapie wurden aufgrund ihrer zunehmenden Bedeutung und Komplexität detaillierter gefasst.

Die Grundprinzipien der Standardtherapie der unkomplizierten Tuberkulose haben sich im Wesentlichen nicht geändert. Die mikrobiologische und radiologische Verlaufsdiagnostik während der Therapie wurde vor allem für die späte Kontinuitätsphase und für die Nachbeobachtung vereinfacht und wird grafisch dargestellt.

Im Kapitel Versorgungsaspekte der Tuberkulose werden die rechtlichen und organisatorischen Aspekte der Tuberkulosebehandlung thematisiert. Praktische Hinweise auch zu ökonomischen Gesichtspunkten sollen die Behandler in Deutschland bei wichtigen Entscheidungen in der Therapieführung unterstützen.

Im Kapitel Labordiagnostik wird auf Aspekte der Präanalytik eingegangen, die für die notwendige Quantität und für die Qualität der Untersuchungsmaterialien entscheidend sind. In den letzten Jahren wurden die molekularbiologische Erregerdiagnostik und Empfindlichkeitsprüfung in Studien validiert und haben dadurch einen höheren Stellenwert erlangt. Die zeitnahe Bestimmung von Resistenzen gegen die Medikamente der Standard- und der NichtStandardtherapie ist eine wichtige Voraussetzung für eine effiziente Behandlung.

Die Risiken, die bei einer latenten tuberkulösen Infektion (LTBI) in Niedrigprävalenzländern die Progression zur Tuberkulose begünstigen, werden auf Grundlage aktueller Studien bewertet. Für Deutschland werden Konsensemp- fehlungen gegeben, welche Personengruppen bei positivem Testergebnis einer präventiven Therapie zugeführt werden sollten.

Resistenzen gegen Tuberkulosemedikamente werden zunehmend zu einem globalen Problem. ${ }^{2}$ In Deutschland ist der Anteil der MDR-Tuberkulosen auf niedrigem Niveau (bei jedoch relevanten Fallzahlen) stabil, die Behandlungserfolge liegen auch in Deutschland weit unter dem von der Weltgesundheitsorganisation (WHO) angestrebten Ziel von $70 \%{ }^{3}$ Daher widmet die aktuelle Leitlinie dem Thema Mehrfachresistenzen und UAW ein eigenes Kapitel.

Die Behandlung von Tuberkulosepatienten mit HIV-Koinfektion ist aufgrund häufiger Interaktionen der Tuberkulosemedikamente - insbesondere der Rifamycine - mit der antiretroviralen Therapie (ART) oft mit Schwierigkeiten verbunden. Daher wird in der Leitlinie detailliert in einem eigenen Kapitel auf die Kombinationsmöglichkeiten und die pharmakologischen Besonderheiten bei gleichzeitiger Therapie von Tuberkulose und HIV-Infektion eingegangen. Die Datenlage zur Bedeutung einer Überwachung der therapeutischen Medikamentenspiegel (engl. TDM - therapeutic drug monitoring) ist derzeit für eine starke Empfehlung noch nicht ausreichend. Wegen ihres Potenzials zur Verbesserung des Therapieerfolges und zur Vermeidung von Medikamenten-Resistenzen wird die Anwendung von TDM in bestimmten Situationen dennoch empfohlen. So kann die Bestimmung der Serumspiegel insbesondere bei HIV-TB-Koinfektion, aber auch in anderen Situationen wie bei Therapieversagen nach zwei bis drei Monaten Behandlung oder bei der Behandlung einer MDR-Tuberkulose helfen, Unter- und Überdosierungen zu erkennen und die Medikation entsprechend anzupassen.

Die Beschreibung der einzelnen Arzneimittel zur Tuberkulosetherapie mit ihren pharmakodynamischen und pharmakokinetischen Besonderheiten wurde grundlegend überarbeitet und erweitert. Die alphabetische Übersicht ermöglicht einen schnellen Überblick auch zum Umgang mit unerwünschten Arzneimittelwirkungen.

\footnotetext{
Literatur

1. Schaberg T, Bauer T, Castell S, et al.: Empfehlungen zur Therapie, Chemoprävention und Chemoprophylaxe der Tuberkulose im Erwachsenenund Kindesalter. Pneumologie 2012;66:133- 171

2. WHO: Global tuberculosis report 2016. Im Internet: www.who.int/tb/publications/global_report/en/

3. RKI: Bericht zur Epidemiologie der Tuberkulose in Deutschland für 2015. Zugriff: 29.1.2017
}

- Dr. Ralf Otto-Knapp

Deutsches Zentralkomitee zur Bekämpfung der Tuberkulose

Korrespondenz: rotto-knapp@dzk-tuberkulose.de

- Vorgeschlagene Zitierweise:

Otto-Knapp R: Neue S2k-Leitlinie zur Tuberkulose im Erwachsenenalter. Epid Bull 2017;11/12:104

DOI 10.17886/EpiBull-2017-013 